\title{
Minimização de Defeitos em Revestimentos de Superligas de Níquel Depositada Pelo Processo TIG com Alimentação de Arame Frio
}

\author{
(Minimization of Defects in Nickel-Based Superalloy Weld Overlay Deposited by the GTAW Cold Wire Feed Process)
}

\author{
Cleiton Carvalho Silva, Edvan Cordeiro de Miranda, Marcelo Ferreira Motta, Hélio Cordeiro de Miranda, \\ Jesualdo Pereira Farias \\ Universidade Federal do Ceará, Departamento de Engenharia Metalúrgica e de Materiais, Laboratório de Engenharia de \\ Soldagem. Campus do Pici, Bloco 750, Amadeu Furtado, CEP: 60455-760, Fortaleza, Ceará. cleiton@metalmat.ufc.br
}

\begin{abstract}
Resumo
O objetivo do presente trabalho foi avaliar a influência dos parâmetros de soldagem na formação de defeitos na soldagem de revestimentos com ligas à base de níquel, e sua possível eliminação através do correto ajuste dos referidos parâmetros. Para tanto, foram depositados revestimentos com as ligas à base de Ni do tipo Inconel 625, Hastelloy C276 e Inconel 686, sobre um substrato de aço C-Mn, através do processo TIG com alimentação de arame frio. O planejamento dos experimentos foi realizado aplicando-se o método Taguchi. Os fatores de controle avaliados foram a Técnica da energia (TE), o nível de energia de soldagem (E), o tipo de liga $(L)$, o gás de proteção $(G)$ e o tipo de tecimento $(T)$. Outros parâmetros foram mantidos constantes, tendo sido investigados previamente. Os resultados mostraram que o tipo de tecimento em espiral, embora contribua significativamente para a redução da diluição, causa uma forte instabilidade ao processo, resultando na maioria dos casos em defeitos superficiais ou defeitos entre passes. A condição ótima para evitar a formação de defeitos entre passes identificada pelo método Taguchi foi constituída pelas seguintes combinação de fatores de controle 2-2-2-3-3, ou seja: TE-I; Emédia; Liga Hastelloy C276; Gás de proteção Ar+He; Tecimento Duplo-8. A condição ótima para a soldagem sem defeitos resulta em alto nível de diluição não sendo indicada para a soldagem de revestimentos resistentes à corrosão.
\end{abstract}

Palavras-chave: soldagem TIG, arame frio, revestimentos, defeitos, ligas de níquel

Abstract: The objective of the present work was to evaluate the influence of the welding parameters in the formation of defects in weld overlays with nickel-based alloys, and its possible elimination through the correct adjustment of the welding parameters. Coatings were deposited with the nickel-based alloys type Inconel 625, Hastelloy C276 and Inconel 686, on C-Mn steel plates, using GTAW cold wire feed process. The planning of the experiments was accomplished being applied the Taguchi method. The control factors evaluated were the Energy Technique (ET), the welding heat input (E), the type of alloy (L), the shielding gas $(G)$ and the type of arc oscillation (T). Other parameters were maintained constant, having previously been investigated. The results showed that the arc oscillation type in spiral, although it contributes significantly to reduce the dilution level, it cause a strong instability to the process, resulting in most of the cases in surface defects or defects among passes. The optimum condition to avoid defects among passes was identified by the Taguchi method, which was constituted by the following combination of control factors: 2-2-2-3-3, or be: I energy technique (TE-I); medium heat input level ( $\left.E_{\text {medium }}\right)$; Hastelloy C276 alloy; Shielding gas Ar+He; Double-8 arc oscillation. The optimum condition for the welding without defects results in high dilution level not being indicated for the welding of resistant coverings to the corrosion.

Keywords: GTAW welding, cold wire, overlay, deffects, nickel-based alloy.

\section{INTRODUÇÃO}

Ligas a base de níquel estão entre os materiais de engenharia mais resistentes à corrosão em uma larga faixa de ambientes, inclusive para aplicações em alta temperatura [1]. O níquel como elemento principal destas ligas, possui propriedades sem

Recebido em 15/09/2014, texto final em 03/11/2014.

DOI: http://dx.doi.org/10.1590/0104-9224/SI1904.05 igual que fazem dele uma base ideal para o desenvolvimento de materiais resistentes à corrosão. Dentre diversas características importantes, destaca-se a sua capacidade de formação de filmes passivos contínuos e que protegem o material contra a ação do ambiente [2]. Outra importante característica do níquel como elemento principal é a sua habilidade em dissolver significativa quantidade de elementos de ligas, mantendo-os em solução sólida, e permitindo inúmeras possibilidades para o desenvolvimento de novas ligas com características e aplicações específicas [3]. Elementos de liga como Cr, Mo e W são exemplos de elementos que introduzem propriedades especiais próprias para aumentar 
a resistência à corrosão. $\mathrm{O} \mathrm{Cr}$ e o Mo são notadamente os mais importantes elementos de liga relacionados ao aumento da resistência à corrosão. Juntos eles parecem apresentar um efeito sinérgico, especialmente em relação à corrosão localizada. No entanto, seu alto custo pode inviabilizar o seu emprego na construção de equipamentos maciços.

Neste contexto, a soldagem de revestimento torna-se uma excelente opção para a fabricação de equipamentos para inúmeros setores industriais, cujas superfícies internas ou externas necessitam de características específicas para garantir um bom desempenho em serviço, como por exemplo, resistência à corrosão [4]. Um dos aspectos mais benéficos da soldagem de revestimento é a redução do custo do equipamento em relação à sua fabricação maciça com material nobre. A formação do revestimento é conseguida através da deposição de uma ou mais camadas, e cada camada é produzida por meio da deposição de múltiplos cordões de solda, dispostos lado a lado. A qualidade de um revestimento obtida através da deposição de camadas de solda sobre um substrato está, em geral, relacionada com a correta escolha dos parâmetros de soldagem, de forma a produzir revestimentos com espessura adequada e com reduzido grau de diluição, especialmente em soldagens dissimilares de ligas que devem apresentar alta resistência à corrosão [5,6]. Recentemente, alguns trabalhos têm apresentado estudos sobre a aplicação de revestimentos por diversos processos, no entanto, nas avaliações são considerados somente os resultados de diluição e características geométricas para apenas um cordão de solda [7-10]. É obvio que o estudo das condições operacionais com simples cordão é capaz de fornecer informações relevantes sobre a diluição, penetração, reforço, etc., além de reduzir o tempo e a quantidade de experimentos. Contudo, é importante destacar que na soldagem multipasses para formação de camadas de revestimentos, também devem ser considerados estudos com múltiplos passes, especialmente para se verificar as condições de sobreposição dos cordões de solda que garantam um bom acabamento superficial e ausência de defeitos.

Infelizmente, a grande quantidade de parâmetros a serem avaliados na busca de condições que possibilitem uma boa camada de revestimento com geometria da solda requerida e boa qualidade constituem um problema comum para os fabricantes de equipamentos e estruturas soldadas. Para superar este problema e tornar menos oneroso o processo de avaliação das condições operacionais em soldagem, vários métodos estatísticos de projeto de experimentos têm sido desenvolvidos para otimização dos parâmetros de entrada em função de uma ou mais características do produto/processo que se deseja alcançar [11]. Dentre os vários métodos de projeto de experimentos, o método Taguchi tem se destacado nos últimos anos no cenário industrial, sendo aplicado no controle e otimização de processos de fabricação como fundição, furação, torneamento, eletrodeposição, dentre outros [12]. Em especial na área de soldagem, o método Taguchi tem sido aplicado não só no estudo das características geométricas, mas também na otimização das propriedades mecânicas [13].

Por ser uma poderosa ferramenta de planejamento de experimentos capaz de indicar os parâmetros ótimos de um processo industrial que resultem na maximização de uma determinada propriedade/característica, como por exemplo, a qualidade do produto, o método Taguchi foi escolhido para ser utilizado na presente investigação. Este método utiliza um arranjo ortogonal planejado especialmente para cada condição a ser avaliada, tornando possível estudar os efeitos dos parâmetros com um reduzido número de experimentos.

Assim, o objetivo do presente trabalho foi otimizar as condições operacionais, não só relacionada aos parâmetros de soldagem, mas também com relação aos consumíveis (material de adição e gases de proteção), utilizando como ferramenta o método Taguchi, visando à deposição de revestimentos sem defeitos e que não comprometam a diluição nem a espessura de camada.

\section{MÉTODO TAGUCHI}

\subsection{Seleção dos Fatores de Controle}

Com base em resultados anteriores foi possível verificar que algumas variáveis influenciam significativamente as características geométricas e a diluição dos cordões de solda [14], necessitando por tanto, de uma avaliação mais detalhada quanto aos seus efeitos sobre a soldagem multipasse propriamente dita. Como foco principal foco é a obtenção de revestimentos com baixo nível de diluição e reforço, mas sem a presença de defeitos, os mesmos parâmetros usados na avaliação da diluição e características geométricas foram aplicados para tentar suprimir qualquer $r$ formação de defeitos. $\mathrm{O}$ tecimento foi um parâmetro que apresentou influência sobre as características geométricas e diluição, representando um importante fator de controle a ser estudado [15]. Por este motivo, foram selecionados três diferentes tipos de tecimento. Quanto à velocidade de alimentação de arame, verificou-se em estudos preliminares que esta variável é diretamente proporcional ao nível de corrente de soldagem, não devendo ser selecionada como variável independente. Além disso, foi verificado tanto em análises anteriores quanto na literatura [16] que a melhor condição em relação à redução da diluição é alcançada quando aplicada a máxima velocidade de alimentação.

Outras variáveis que tiveram efeito moderado ou cujos resultados anteriores indicaram valores ótimos para a aplicação dos revestimentos foram mantidas fixas no presente estudo. A Distância da Ponta do Eletrodo à Peça (DPEP) de $10 \mathrm{~mm}$ foi escolhida por resultar em baixos níveis de diluição [17]. Já a Distância da Ponta do Arame à Peça (DPAP) foi fixada em 3 $\mathrm{mm}$ por também apresentar uma boa relação entre geometria e diluição [18].

\subsection{Seleção da Matriz Ortogonal}

A seleção do arranjo ortogonal mais adequado para viabilizar o estudo das variáveis deve levar em conta o número de fatores de controle e o número de níveis de cada fator, além de possíveis interações entre os fatores, sendo todos estes associados ao grau de liberdade total dos experimentos [19]. Os graus de liberdade correspondem à quantidade de comparações independentes que podem ser realizadas dentro do universo de dados disponíveis. 
O número de graus de liberdade de um fator é definido como o número de níveis do fator menos um $(\mathrm{n}-1)$. Para a correta escolha do arranjo ortogonal, deve-se garantir que os graus de liberdade do arranjo sejam maiores ou pelo menos igual aos graus de liberdade dos parâmetros estudados (fatores de controle). Visto que no presente estudo foram selecionados cinco fatores de controle, sendo um com dois níveis (TE) e os demais $(E, G, L, T)$ com três níveis, têm-se então um total de 9 graus de liberdade.

Com base somente no grau de liberdade, os arranjos possíveis seriam o L9, L12 e L16 que atenderiam as necessidades quanto aos graus de liberdade dos experimentos, porém, estes só permitem o uso de fatores de controle com dois níveis. Devido a isso, optou-se por um arranjo ortogonal do tipo L18, conforme mostrado na Tabela 1, o qual apresenta uma coluna com dois níveis e quatro colunas com três níveis, permitindo assim o estudo de todos os fatores de controle escolhidos, e também atende as exigências quanto aos graus de liberdade dos experimentos. Nesta tabela também são apresentados os valores de corrente de soldagem, velocidade de soldagem, tensão de soldagem, energia de soldagem e velocidade de alimentação (valor médio dos cordões depositados). A designação de cada condição de soldagem testada é dada pela letra $\mathrm{T}$ de Taguchi, seguido do número correspondente à linha da Tabela 1 . A descrição dos níveis para cada fator de controle encontra-se na Tabela 2.

\subsection{Razão sinal ruído $\mathrm{S} / \mathrm{R}$}

No método Taguchi, a característica de qualidade que se pretende analisar é medida através da determinação da razão sinal/ruído ( $\mathrm{S} / \mathrm{N}$ - sinal/noise), também denominada de $\eta$. Nesta razão o termo sinal corresponde ao valor médio da característica estudada e o ruído refere-se ao quadrado do desvio padrão $\sigma^{2}$ (variância).

Tabela 1. Parâmetros de soldagem referente à planilha L18 gerada pelo Método Taguchi.

\begin{tabular}{|c|c|c|c|c|c|c|c|c|c|c|}
\hline Ensaio & TE & $\mathbf{E}$ & $\mathbf{L}$ & $\mathbf{G}$ & $\mathbf{T}$ & $\begin{array}{c}\text { Corrente } \\
\text { Eficaz I } \mathbf{( A )}\end{array}$ & $\begin{array}{c}\text { Velocidade de } \\
\text { soldagem V } \\
\mathbf{( c m / m i n )}\end{array}$ & $\begin{array}{c}\text { Tensão Eficaz } \\
\mathbf{U} \text { (V) }\end{array}$ & $\begin{array}{c}\text { Energia de } \\
\text { soldagem } \\
\mathbf{( k J / c m})\end{array}$ & $\begin{array}{c}\text { Velocidade de } \\
\text { alimentação de } \\
\text { arame (m/min) }\end{array}$ \\
\hline $\mathrm{T} 1$ & 1 & 1 & 1 & 1 & 1 & 334,2 & 25 & 21,2 & 17,0 & 6,0 \\
\hline $\mathrm{T} 2$ & 1 & 1 & 2 & 2 & 2 & 334,2 & 25 & 20,9 & 16,8 & 6,0 \\
\hline $\mathrm{T} 3$ & 1 & 1 & 3 & 3 & 3 & 335,5 & 25 & 23,9 & 19,3 & 8,5 \\
\hline $\mathrm{T} 4$ & 1 & 2 & 1 & 1 & 2 & 334,3 & 21 & 20,2 & 19,3 & 7,0 \\
\hline $\mathrm{T} 5$ & 1 & 2 & 2 & 2 & 3 & 334,4 & 21 & 20,8 & 19,9 & 7,0 \\
\hline $\mathrm{T} 6$ & 1 & 2 & 3 & 3 & 1 & 336,9 & 21 & 22,9 & 22,1 & 9,5 \\
\hline $\mathrm{T} 7$ & 1 & 3 & 1 & 2 & 1 & 334,6 & 17 & 20,5 & 24,2 & 8,0 \\
\hline $\mathrm{T} 8$ & 1 & 3 & 2 & 3 & 2 & 335,4 & 17 & 21,8 & 25,8 & 11,0 \\
\hline $\mathrm{T} 9$ & 1 & 3 & 3 & 1 & 3 & 334,3 & 17 & 20,1 & 23,7 & 8,0 \\
\hline $\mathrm{T} 10$ & 2 & 1 & 1 & 3 & 3 & 285,8 & 21 & 21,5 & 17,6 & 8,5 \\
\hline $\mathrm{T} 11$ & 2 & 1 & 2 & 1 & 1 & 285,1 & 21 & 20,2 & 16,5 & 6,0 \\
\hline $\mathrm{T} 12$ & 2 & 1 & 3 & 2 & 2 & 283,3 & 21 & 19,7 & 16,0 & 6,0 \\
\hline $\mathrm{T} 13$ & 2 & 2 & 1 & 2 & 3 & 333,5 & 21 & 21,0 & 20,0 & 7,0 \\
\hline $\mathrm{T} 14$ & 2 & 2 & 2 & 3 & 1 & 335,4 & 21 & 23,6 & 22,6 & 9,5 \\
\hline $\mathrm{T} 15$ & 2 & 2 & 3 & 1 & 2 & 333,6 & 21 & 20,7 & 19,7 & 7,0 \\
\hline $\mathrm{T} 16$ & 2 & 3 & 1 & 3 & 2 & 386,8 & 21 & 23,4 & 25,9 & 11,0 \\
\hline $\mathrm{T} 17$ & 2 & 3 & 2 & 1 & 3 & 384,8 & 21 & 21,4 & 23,5 & 8,5 \\
\hline $\mathrm{T} 18$ & 2 & 3 & 3 & 2 & 1 & 383,6 & 21 & 21,2 & 23,2 & 8,5 \\
\hline
\end{tabular}

Tabela 2. Designação dos fatores de controle para o Método Taguchi.

\begin{tabular}{|c|c|c|c|}
\hline \multirow{2}{*}{ Fatores de controle } & \multicolumn{3}{|c|}{ Níveis } \\
\cline { 2 - 4 } & $\mathbf{1}$ & $\mathbf{2}$ & $\mathbf{3}$ \\
\hline Técnica da energia (TE) & TE V & TE I & Alta \\
\hline Energia de soldagem (E) & Baixa & Média & Inconel 686 \\
\hline Liga (L) & Inconel 625 & Hastelloy C-276 & Ar + He \\
\hline Gás de Proteção (G) & Ar puro & Ar + NO & Duplo 8 \\
\hline Tecimento (T) & Espiral & Triangular & \\
\hline
\end{tabular}


Os resultados são otimizados de acordo com a categoria na qual a característica da qualidade melhor se adéqua, podendo ser: menor-melhor (Eq. 1), maior-melhor (Eq. 2) e nominalmelhor (Eq. 3), conforme as equações apresentadas a seguir [19]. No caso de revestimentos, tem-se como objetivo produzir uma camada com baixa penetração que resulte num baixo nível de diluição, com reforço adequado (ou seja, superior a $3 \mathrm{~mm}$ ) e com ausência de defeitos. Obviamente que não será possível otimizar todas as características de qualidade, ou variável resposta, listadas, sendo necessário então avaliar qual ou quais destas são primordiais para a aplicação que se deseja.

$$
\begin{array}{ll}
\eta=-10 \log _{10}\left(\frac{1}{n} \sum_{i=1}^{n} y_{i}^{2}\right) & \text { (Menor melhor) } \\
\eta=-10 \log _{10}\left(\frac{1}{n} \sum_{i=1}^{n} \frac{1}{y_{i}^{2}}\right) & \text { (Maior melhor) }
\end{array}
$$

$$
\eta=10 \log _{10}\left(\frac{\mu^{2}}{\sigma^{2}}\right) \quad \text { (Nominal melhor) }
$$

Onde: $\mu$ é a média dos dados observados, $\sigma$ é o desvio padrão dos dados observados, $n$ é o número de observações e $y_{i}$ é o valor do dado observado para a i-ésima característica de qualidade.
Considerando as características de qualidade: diluição, penetração e defeitos; tem-se que tais variáveis resposta devem ser classificadas na categoria menor-melhor, tendo em vista os requisitos exigidos para revestimentos - especialmente baixa diluição e ausência de defeitos. No entanto, o reforço deve ser agrupado na categoria maior melhor, visto que quanto maior o reforço, maior será a espessura da camada depositada e, consequentemente, menor será a necessidade de deposição de múltiplas camadas.

\section{MATERIAIS E MÉTODOS}

Para a realização deste trabalho foram empregados diversos materiais, denominados de consumíveis de soldagem e classificados em eletrodo, metais de adição, metais de base e gases de proteção. O eletrodo de tungstênio utilizado no processo TIG foi do tipo toriado, com diâmetro de 4,0 mm. Os metais de adição foram as ligas AWS ER-NiCrMo-3 (Inconel 625), AWS ER-NiCrMo-4 (Hasteloy C-276) e AWS ER$\mathrm{NiCrMo-14}$ (Inconel 686). Todas as ligas foram adquiridas na forma de bobinas com diâmetro de arame de $1,12 \mathrm{~mm}$. O metal de base utilizado foi o aço ASTM A516 Gr 60. As composições químicas destes materiais encontram-se na Tabela 3. Os gases de proteção utilizados foram argônio puro (99,99\%), argônio com

\begin{tabular}{|c|c|c|c|c|c|c|c|c|}
\hline Item & \multicolumn{8}{|c|}{ Composição, \% em peso } \\
\hline \multirow{4}{*}{$\begin{array}{l}\text { AWS ERNiCrMo-3 } \\
\text { (INCONEL 625) }\end{array}$} & $\mathbf{N i}$ & C & $\mathrm{Cr}$ & Mo & W & $\mathrm{Fe}$ & Al & $\mathbf{T i}$ \\
\hline & 64,43 & 0,011 & 22,2 & 9,13 & - & 0,19 & 0,09 & 0,23 \\
\hline & $\mathbf{N b}$ & Mn & $\mathbf{S i}$ & $\mathbf{C u}$ & Co & $\mathbf{V}$ & $\mathbf{P}$ & $\mathbf{S}$ \\
\hline & 3,53 & 0,01 & 0,05 & 0,01 & 0,03 & - & 0,002 & 0,002 \\
\hline \multirow{4}{*}{$\begin{array}{l}\text { AWS ERNiCrMo-4 } \\
\text { (HASTELOY C-276) }\end{array}$} & $\mathbf{N i}$ & $\mathrm{C}$ & $\mathrm{Cr}$ & Mo & $\mathbf{W}$ & $\mathbf{F e}$ & $\mathbf{A l}$ & Ti \\
\hline & 56,80 & 0,002 & 16,13 & 16,28 & 3,38 & 6,07 & - & - \\
\hline & $\mathbf{N b}$ & Mn & Si & $\mathrm{Cu}$ & Co & $\mathbf{V}$ & $\mathbf{P}$ & $\mathbf{S}$ \\
\hline & - & 0,52 & 0,03 & 0,06 & 0,13 & 0,17 & 0,07 & 0,002 \\
\hline \multirow{4}{*}{$\begin{array}{l}\text { AWS ERNiCrMo-14 } \\
\text { (INCONEL 686) }\end{array}$} & $\mathbf{N i}$ & $\mathbf{C}$ & $\mathrm{Cr}$ & Mo & $\mathbf{W}$ & $\mathbf{F e}$ & $\mathrm{Al}$ & Ti \\
\hline & 58,22 & 0,01 & 20,53 & 16,39 & 3,97 & 0,29 & 0,26 & 0,04 \\
\hline & $\mathbf{N b}$ & Mn & $\mathbf{S i}$ & $\mathbf{C u}$ & Co & $\mathbf{V}$ & $\mathbf{P}$ & $S$ \\
\hline & - & 0,23 & 0,059 & 0,01 & - & - & 0,002 & 0,001 \\
\hline \multirow{2}{*}{ ASTM A516 Gr. 60} & $\mathbf{N i}$ & $\mathrm{C}$ & $\mathrm{Cr}$ & Mo & $\mathrm{Fe}$ & Al & Mn & $\begin{array}{l}\mathbf{S i} \\
\end{array}$ \\
\hline & 0,01 & 0,15 & 0,02 & 0,01 & Bal. & 0,02 & 0,95 & 0,2 \\
\hline
\end{tabular}
$30 \%$ de hélio e argônio com $0,01 \%$ de NO.

Tabela 3. Composição química dos metais de adição fornecida pelos fabricantes.

A soldagem dos revestimentos foi realizada pelo processo TIG com alimentação de arame frio, operando com corrente contínua constante. A deposição das camadas foi realizada sobre as chapas de aço ASTM A516 Gr. 60 com 200 x 150 x 12,5 $\mathrm{mm}$. O comprimento do revestimento foi de $180 \mathrm{~mm}$ e a largura da camada depositada foi superior à $55 \mathrm{~mm}$. Para garantir a largura mínima da camada foram depositados de 4 a 7 cordões dispostos lado a lado, cuja sobreposição deve ser suficiente para proporcionar um bom acabamento superficial e ausência de defeitos. Todas as chapas foram previamente identificadas e passaram por processo de limpeza superficial por meio de escovamento com escova rotativa de aço inoxidável e limpeza com acetona. Alguns parâmetros foram previamente estudados e mantidos constantes. $\mathrm{O}$ modo de alimentação de arame foi à frente da tocha com o arame passando por dentro do arco. $\mathrm{O}$ ângulo de alimentação do arame foi de $50^{\circ}$, a distância da ponta do arame à peça (DPAP) foi de $3 \mathrm{~mm}$, a distância da ponta do eletrodo à peça (DPEP) de $10 \mathrm{~mm}$, o ângulo de afiação do eletrodo de $50^{\circ}$ e a vazão de gás de $15 \mathrm{l} / \mathrm{min}$.

Antes de realizar a deposição das camadas foi escolhida uma condição de soldagem e realizados testes para determinar alguns parâmetros de tecimento como passo (distância entre cordões), 

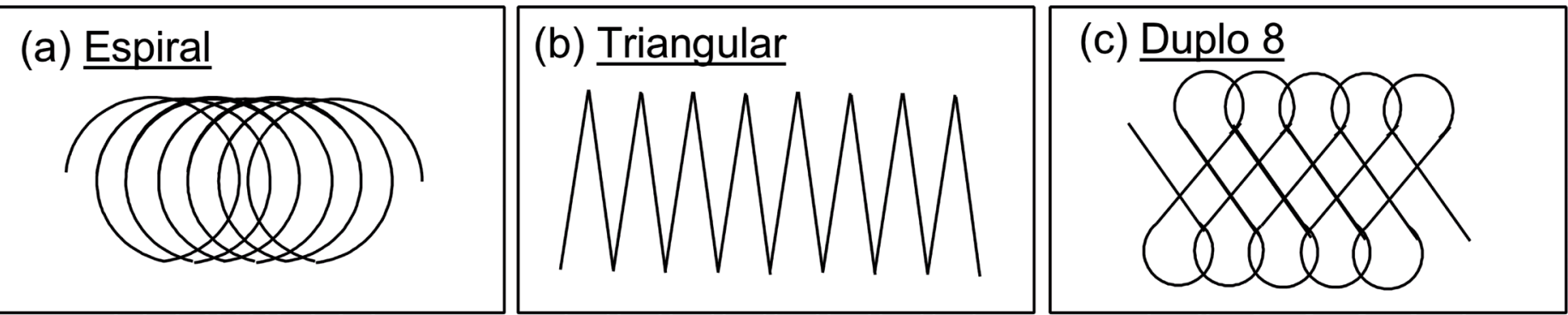

Figura 1. Desenho ilustrando a trajetória descrita para cada tipo de tecimento aplicado no Método Taguchi.

amplitude de oscilação e comprimento de onda. Com base nestes ensaios foi estabelecido como critério o valor do passo correspondente à $2 / 3$ da largura do $1^{\circ}$ cordão de solda depositado. Os demais parâmetros de tecimento são a amplitude de oscilação $-8 \mathrm{~mm}$ e comprimento de onda $-1,2 \mathrm{~mm}$. Outro importante parâmetro avaliado foi o tipo de tecimento descrito pelo arco, o qual foi executado pelo robô. Foram selecionados três tipos de movimento cuja trajetória está predefinida no programa do robô: espiral, triangular, duplo 8. As trajetórias dos tecimentos são ilustradas na Figura 1. Após a deposição por soldagem das camadas, as chapas foram fotografadas, escovadas, pesadas e submetidas a ensaio de líquido penetrante para verificar a possível formação de trincas superficiais. Posteriormente foram retiradas amostras da seção transversal para avaliar as características geométricas dos revestimentos.

\section{RESULTADOS E DISCUSSÃO}

\subsection{Acabamento Superficial}

A primeira avaliação realizada nos corpos de prova definitivos da soldagem dissimilar entre o metal de base ASTM A516 Gr. 60 e a liga de níquel AWS ER NiCrMo-3 (Inconel 625) foi fundamentada na qualidade de sobreposição entre os cordões de solda. Embora esta avaliação tenha sido de caráter subjetivo, sua execução permitiu avaliar o nível do acabamento superficial resultante da camada depositada, considerando principalmente a planicidade, rugosidade e presença de defeitos. A Figura 2 mostra a superfície dos cordões de solda para as quatro condições de soldagem aplicadas nesta etapa.

De uma forma geral, todas as condições de soldagem empregadas apresentaram excelente acabamento superficial. As exceções foram as condições T1, T7, T11 e T 12, as quais não foram capazes de realizar a deposição da camada. Tal resultado foi considerado positivo e reforça as possibilidades de aplicação da maioria destas condições de soldagem em aplicações de revestimento.

Conforme já destacado, nem todas as condições de soldagem empregadas no projeto de experimentos robusto (análise de Taguchi) apresentaram-se aptas para a realização de camadas. A amostra T1 (Figura 2a), soldada com a liga Inconel 625, gás de proteção Ar puro, tecimento espiral, e baixo aporte térmico, não foi capaz de promover a correta sobreposição da camada. Verificou-se que logo após iniciar a soldagem do segundo cordão, houve uma grande oscilação do arco com o tecimento, cujo resultado foi um desvio do arco, o qual não conseguiu em alguns pontos deslocar-se continuamente ao longo da oscilação (tecimento) durante a execução da soldagem, mas sim permanecendo em alguns momentos somente sobre o cordão anterior ou sobre a peça.

Tal comportamento resultou no aspecto do cordão de solda mostrado na Figura 2a. Nesta figura é possível observar inicialmente alguns defeitos causados pela permanência do arco sobre a peça, o qual não conseguiu sobrepor o cordão e preencher a região entre passes. Mais ao final do processo uma porção na qual a deposição ocorreu exclusivamente sobre o cordão de solda anterior também foi observada. Vale salientar que os parâmetros de tecimento e o passo de sobreposição aplicados em todas as condições de soldagem avaliadas na etapa da análise de Taguchi foram iguais, sendo o passo de 2/3 da largura do cordão inicial, escolhido com base em análises anteriores, conforme estudo exploratório.

Os corpos de prova soldados com as condições T2 a T6 conseguiram formar com sucesso a camada, não sendo observada nenhuma falha ou defeito, e apresentando um excelente acabamento superficial (Figura 2b). A amostra soldada com a condição T7 não foi capaz de promover a formação da camada. Como pode ser observado na Figura 2c, inúmeros defeitos foram formados durante a soldagem. Diferentemente do que ocorreu com a amostra T1, nesta condição (T7) o comportamento do arco para formação de defeitos caracterizou-se por sua permanência somente sobre a superfície da chapa, não conseguindo em alguns momentos preencher com o metal líquido a região entre passes, resultando assim nos defeitos observados.

Uma questão intrigante na soldagem de camada com a técnica de energia TE $\mathrm{V}$, no qual a corrente de soldagem permanece constante e a energia de soldagem é variada pela mudança de velocidade de soldagem, é que a ocorrência de defeitos foi observada para as condições de baixa energia de soldagem (T1) e alta energia (T7). Dois fatores de controle são inerentes às condições T1 e T7, e podem estar associados à causa dos defeitos. A primeira é que ambas as condições foram soldadas com tecimento espiral. A segunda observação é que tanto T1 quanto T7, usam gases de proteção com menor tensão de ionização do arco (Ar puro e Ar+NO). Contudo, uma terceira condição soldada agora com TE I, apresentou problemas na soldagem com o tecimento espiral, sendo a condição T11.

Uma terceira condição na soldagem com TE I (soldada com nível de energia intermediário) também utilizou o tecimento espiral (T6), porém, o gás de proteção utilizado foi a mistura Ar+He, a qual apresenta um maior potencial de ionização, aumentando o calor no arco e resultando num metal líquido 

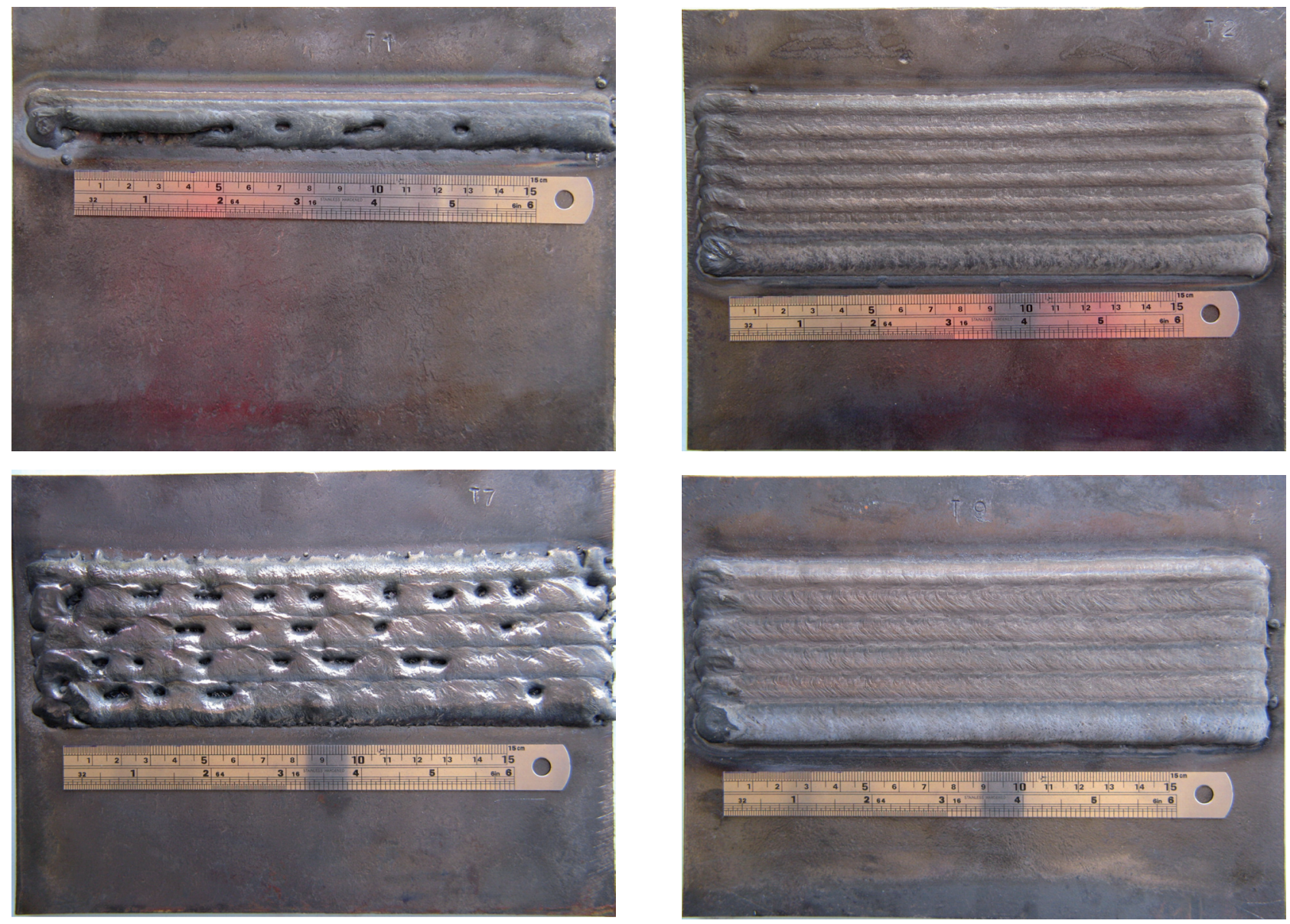

Figura 2. Acabamento superficial dos revestimentos. (a) condição T1; (b) condição T2; (c) condição T7; (d) condição T9.

mais quente, com melhor fluidez, menor tensão superficial, o que melhora a molhabilidade. Com isso, pode-se verificar que os efeitos benéficos associados à adição do hélio no gás de proteção eliminam a possibilidade de defeitos superficiais, tornando a condição com tecimento espiral viável do ponto de vista de defeitos. Destaca-se também que os demais tipos de tecimento (triangular e duplo 8) não apresentaram problemas quando soldados com o Ar puro ou Ar + NO.

Os resultados quanto à técnica de energia TE V (variando o nível de corrente) são apresentados a seguir. Na soldagem com baixo aporte térmico, duas das três condições avaliadas não conseguiram produzir a camada. A condição T10, cujo tecimento foi o duplo 8 e o gás de proteção usado foi a mistura Ar+He, foi a única condição de baixa energia capaz de executar a deposição da camada. Novamente pode ser destacado o papel do gás de proteção na melhoria das propriedades do metal líquido, o qual tem sido extremamente benéfico do ponto de vista de deposição e eliminação de defeitos superficiais.

Destaca-se que o aspecto superficial do cordão de solda para a condição T11 foi semelhante ao produzido pela condição T1, não sendo possível a deposição adequada de uma camada. Assim, as condições de soldagem não foram suficientes para fusão do metal de base e coalescência do metal líquido proveniente da fusão do arame e consequente formação do cordão de solda.

Alguns fatores podem ser decisivos para justificar tal comportamento. Atribui-se tal efeito ao baixo nível de corrente empregado, o qual não fora suficientemente alto para fundir o arame e a peça. Além disso, o gás de proteção usado nesta condição foi Ar puro, que proporciona um arco com menor energia ou mais "frio". Outro fator que pode estar associado é o tipo de tecimento, no caso, espiral, cujo efeito sobre a formação de defeitos já foi identificado anteriormente para as condições TE I.

Todos estes fatores podem atuar em conjunto, prejudicando a soldagem. O baixo nível de corrente de soldagem juntamente com o arco mais frio devido ao uso do Ar permite a fusão do arame, mas não do metal de base. A menor quantidade de calor na soldagem com argônio puro faz com que as gotas de metal líquido tenham alta tensão superficial, não permitindo o molhamento sobre a chapa, nem a continuidade do cordão de solda.

Quanto ao tecimento espiral, este por sua vez, tem como característica uma grande movimentação da poça de fusão, deslocando-a constantemente para a parte fria da chapa, fazendo com que houvesse uma maior perda de calor, se comparado aos tecimentos triangular e duplo 8 , cujo deslocamento à frente é menor ou há inclusive um retrocesso parcial da poça de fusão, evitando perdas excessivas de calor.

A condição T12 também não conseguiu a deposição da camada sobre o substrato de aço carbono. Diferentemente do observado para a amostra T11, na soldagem com a condição T12 houve a completa coalescência entre o metal de adição 
e o metal de base, formando inicialmente um cordão com bom acabamento. Contudo, na sobreposição de passes, assim como observado para os cordões T1 e T7, houve a formação de um cordão descontínuo com a deposição de metal sobre a chapa e sobre a borda do cordão anterior, mas sem promover o preenchimento na região entre passes. Novamente, o baixo aporte térmico deve ser o principal responsável pela presença de defeitos neste caso.

$\mathrm{O}$ gás de proteção utilizado nesta condição $(\mathrm{Ar}+\mathrm{NO})$ também deve favorecer a promoção dos defeitos, haja vista que este também proporciona um arco com menor energia, com isso a resistência elétrica do arco é menor, refletindo assim, em um menor calor do arco. Contudo, destaca-se que em relação ao Ar puro houve uma evolução, visto que a condição T12 foi capaz de promover a soldagem de pelo menos 3 cordões enquanto que na soldagem com Ar puro (T11) não foi possível nem a formação completa de um cordão de solda. Isto pode ser devido à presença em pequena quantidade de gás ativo (óxido nítrico NO), o qual deve atuar na diminuição da tensão superficial. Este comportamento de redução da tensão superficial pela presença de gás ativo em aços tem sido reportado na literatura. Efeito semelhante deve ocorrer para o níquel. Quanto ao tecimento na condição T12 (triangular), este é menos prejudicial para a perda de calor, pois o arco avança menos, concentrando mais o calor.

As demais camadas soldadas com as condições de energia de soldagem média (T13 a T15) e energia de soldagem alta (T16 a T18) não apresentaram defeitos superficiais. A ausência de defeitos superficiais para estas condições foi atribuída ao maior nível de corrente de soldagem empregada, a qual aumenta a potência e, consequentemente, a energia de soldagem, favorecendo principalmente uma melhor fusão do arame e um metal líquido mais quente, com menor viscosidade e tensão superficial, o que facilita a molhabilidade, a formação e a sobreposição dos cordões de solda.

\subsection{Defeitos entre passes}

Os resultados da avaliação das características geométricas e da diluição obtidas através da seção transversal das camadas depositadas foram avaliados em um trabalho anterior, não sendo diretamente o escopo do presente trabalho, cujo foco avaliar a influência dos parâmetros de soldagem na formação de defeitos na soldagem de revestimentos com ligas à base de níquel, e sua possível eliminação através do correto ajuste dos referidos parâmetros. Maiores detalhes sobre a avaliação das características geométricas podem ser obtidas em Silva et al. [5].

Além dos defeitos superficiais apresentados no item anterior, outra característica de qualidade a ser considerada na análise das camadas depositadas, é a ausência de defeitos na região entre passes, como podem ser observados nas Figuras 3 e 4. Recentemente, alguns trabalhos têm sido encontrados na literatura apresentando estudos sobre a aplicação de revestimentos por diversos processos, no entanto, tais trabalhos avaliam somente as características geométricas e diluição através da deposição de apenas um cordão de solda [7-10].

Embora o estudo das condições operacionais através da deposição de um único cordão de solda seja capaz de fornecer informações importantes com relação à diluição e as demais características geométricas (penetração, largura e reforço), além de reduzir o tempo e a quantidade de experimentos. Contudo, na soldagem multipasse para a formação da camada de revestimento também deve ser considerado no estudo a interação entre cordões, especialmente para se verificar as condições de sobreposição que venham a garantir um bom acabamento superficial. No caso da soldagem TIG com alimentação de arame frio, a instabilidade que pode ocorrer devido ao fato da alimentação do arame ser independente do arco voltaico, e a influência da quantidade de calor usada para a fusão do arame, altera as características físicas do metal líquido, tornando ainda mais importante a avaliação através da deposição de camadas.

O Ensaio T1, embora tenha depositado somente dois cordões de solda, apresentou grande instabilidade, a qual resultou tanto em defeitos superficiais quanto na formação de grandes defeitos entre passes. Assim, foram atribuídos 6 defeitos a estes ensaios, que correspondem a provável quantidade de interseções que haveriam entre os passes e, portanto, correspondem a estimativa de defeitos que deveriam ser observados na análise da seção transversal, já que não foi possível soldar esta condição até o
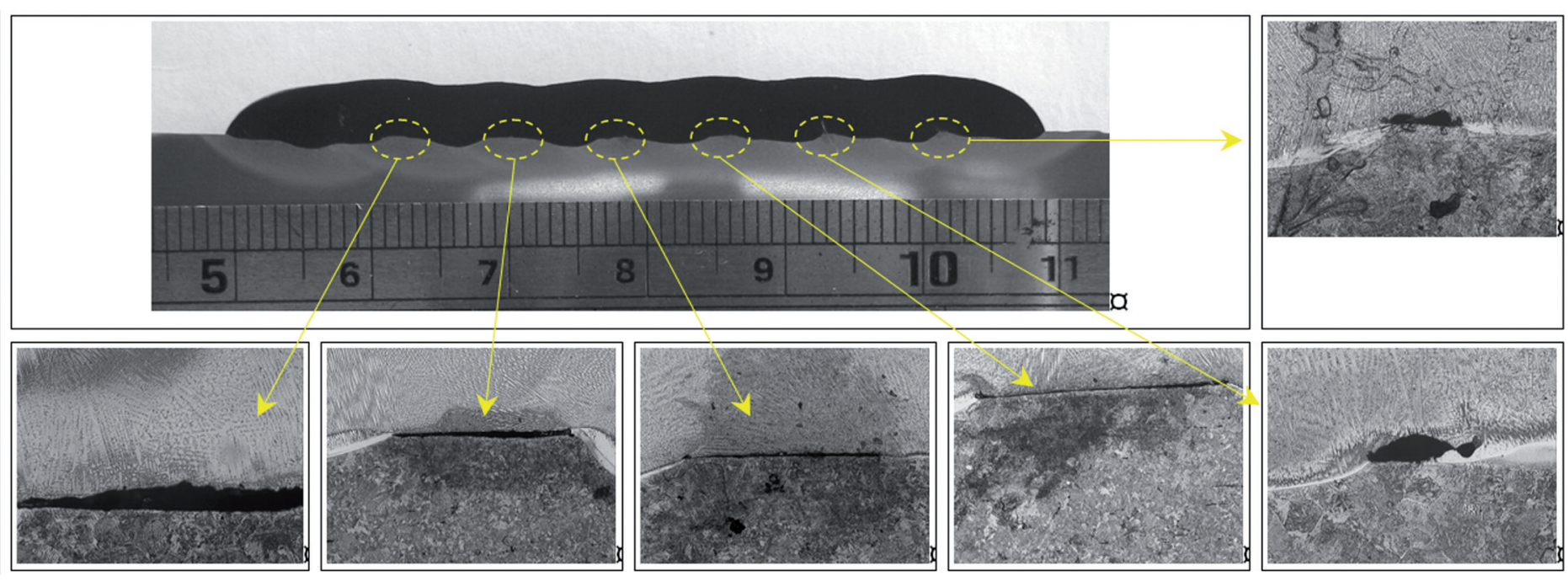

Figura 3. Detalhe dos defeitos entre passes para a amostra T4. 
fim em virtude de sua baixa qualidade. Para as demais condições que não soldaram adequadamente - T7, T11 e T12 - também foram atribuídos 6 defeitos, sendo que a condição T7, a qual optou-se por soldar até atingir a largura necessária, comprovou as suspeitas, apresentando defeitos em todas as regiões entre passes.

Dentre as condições que conseguiram depositar sem promover defeitos superficiais, verificou-se que o ensaio T4 apresentou defeitos em todas as regiões entre passes. As seções transversais das amostras T8 e T9, ambas soldadas com alta energia para a técnica TE V, também apresentaram defeitos entre passes. Além destas a condição T7, que também havia apresentado defeitos superficiais, e que também compõe o grupo TE V com alta energia, apresentaram conforme já destacado defeito em todas as interseções entre os cordões. Em comum, todas apresentaram alta instabilidade e formação de defeitos, indicando que o uso de alta energia para a técnica TE V não é uma condição favorável à deposição de camada.

A condição T10 também apresentou defeitos entre passes. Uma observação quanto ao grupo de ensaios que esta amostra está vinculada, é que as demais amostras do grupo (T11 e T12) não conseguiram executar a deposição adequada da camada, com defeitos superficiais ou até mesmo sem conseguir a coalescência entre o metal de adição e o substrato (T11). A amostra T15 apresentou apenas dois defeitos na região entre passes. Contudo, para aplicações em que se necessita alta confiabilidade a ausência total de defeitos deve ser considerada fundamental para garantia da integridade estrutural dos equipamentos e dutos. A condição T18 embora tenha alcançado excelentes níveis de diluição, abaixo de 5\%, não conseguiu evitar a presença de defeitos. A quantidade de defeitos em cada condição da matriz de experimentos está apresentada na Tabela 4.

\subsection{Análise pelo método Taguchi}

Para avaliar o efeito dos fatores de controle sobre a formação dos defeitos entre os passes utilizou-se a classificação menormelhor, no entanto, durante a condução da análise verificouse uma inconsistência devido ao preenchimento da matriz experimentos com resultados com ausência de defeitos (zero defeitos), que resultaram em erro na determinação do valor do $\eta$. Em decorrência deste fato, foi estabelecido ao invés do valor absoluto da quantidade de defeitos, um valor referente a faixas, sendo o valor 2 atribuído às amostras que não apresentaram defeitos, 4 para as amostras que apresentaram de 2 a 3 defeitos, 8 para amostras com 4 ou 5 defeitos, 10 para amostra com quantidade de defeitos maior que 5. Tal medida possibilitou a análise dos resultados.

O gráfico de Taguchi (Figura 5) mostra que em termos de defeitos, a técnica da energia não teve efeito. Quanto ao nível de energia de soldagem empregado, verificou-se que o nível intermediário de aporte térmico otimizou significativamente o comportamento da formação de defeitos. Este resultado é importante, pois demonstra que o uso da energia no nível intermediário aplica-se tanto à otimização da diluição quanto ao combate sobre a formação de defeitos entre os passes que compõem o revestimento [5].
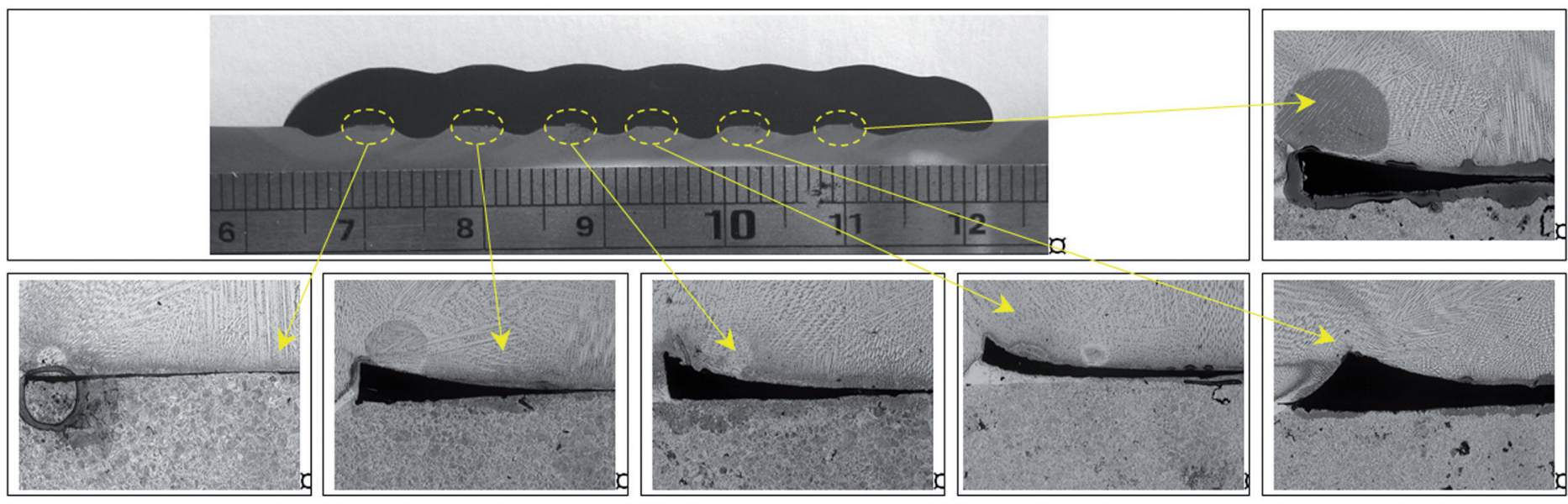

Figura 4. Detalhe dos defeitos entre passes para a amostra T10.

Tabela 4. Quantidade de defeitos entre passes.

\begin{tabular}{|c|c|c|c|c|c|}
\hline Condição & Quantidade de defeitos & Condição & Quantidade de defeitos & Condição & Quantidade de defeitos \\
\hline $\mathrm{T} 1$ & 6 & $\mathrm{~T} 7$ & 6 & $\mathrm{~T} 13$ & 0 \\
\hline $\mathrm{T} 2$ & 0 & $\mathrm{~T} 8$ & 3 & $\mathrm{~T} 14$ & 0 \\
\hline $\mathrm{T} 3$ & 0 & $\mathrm{~T} 9$ & 5 & $\mathrm{~T} 15$ & 2 \\
\hline $\mathrm{T} 4$ & 6 & $\mathrm{~T} 10$ & 6 & $\mathrm{~T} 16$ & 0 \\
\hline $\mathrm{T} 5$ & 0 & $\mathrm{~T} 11$ & 6 & $\mathrm{~T} 17$ & 0 \\
\hline $\mathrm{T} 6$ & 0 & $\mathrm{~T} 12$ & 6 & $\mathrm{~T} 18$ & 5 \\
\hline
\end{tabular}


Influência dos fatores de controle sobre a quantidade de defeitos

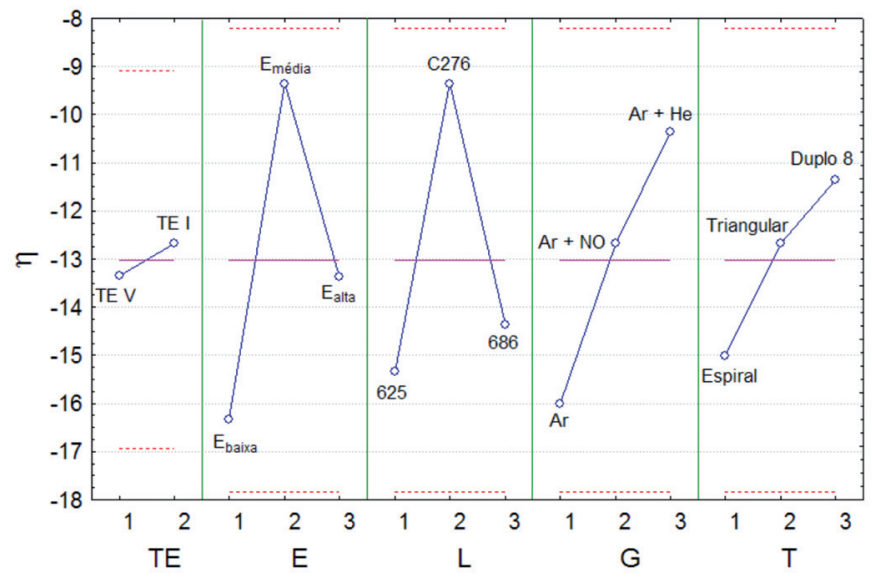

Figura 5. Gráfico de Taguchi para os defeitos entre passes. TE Técnica da energia; E - Energia de soldagem; L - tipo de liga; $\mathrm{G}$ - Gás de proteção; T - Tecimento.

Dentre as ligas avaliadas, a liga L2 (Hastelloy C276) foi a que apresentou melhor resposta à formação de defeitos. Este comportamento tem sua explicação atribuída a algumas características físicas no estado líquido como viscosidade, fluidez, tensão superficial, dentre outras. Embora todas as ligas sejam à base de níquel, é possível que a alterações na composição química possam causar modificações nestas propriedades. Assim, com uma menor viscosidade e maior fluidez, o metal líquido da liga L2 tem maior capacidade para preencher as regiões entre os passes. Por outro lado, estas propriedades contribuem negativamente para o reforço e a diluição [5].

O gás de proteção que mais agregou benefícios ao processo quanto à formação de defeitos nos revestimentos foi a mistura $\mathrm{Ar}+\mathrm{He}$, visto que o maior calor do arco proporciona não só uma maior quantidade de material fundido, mas também um metal líquido mais quente que apresenta menor viscosidade e maior fluidez. No entanto, é importante destacar o comportamento da mistura $\mathrm{Ar}+\mathrm{NO}$, a qual otimizou significativamente o $\eta$, em relação ao Ar puro. Tal efeito, embora não esperado visto que tal gás foi elaborado inicialmente por questões ambientais [20,21], pode ser atribuído a uma possível atuação do gás ativo presente nesta mistura (NO) sobre a diminuição da tensão superficial do metal líquido, a qual resulta em um aumento da fluidez e da molhabilidade do cordão, implicando assim no preenchimento da região entre passes pelo metal líquido. Além disso, este gás também resultou em boas características geométricas e baixo nível de diluição [5], indicando se tratar de uma boa opção para a soldagem de revestimentos com este tipo de liga.

Quanto ao tipo de tecimento, verificou-se uma grande contribuição do tecimento tipo duplo 8 em relação aos demais. Neste tipo de tecimento o arco descreve uma trajetória em forma de hélice que faz com que o arco avance pouco para frente, fazendo com que haja uma menor perda de calor, se comparado aos tecimentos triangular e espiral, cujo deslocamento à frente é maior, evitando perdas excessivas de calor e melhorando o escoamento do metal líquido na borda do cordão anterior.

Embora o método Taguchi tenha se mostrado uma boa opção para indicar os parâmetros ótimos que resultam na ausência ou redução de defeitos, tais combinações de parâmetros são antagônicas se comparadas às condições ótimas para a diluição, desfavorecendo tal característica de qualidade, a qual resulta em prejuízos para as propriedades do revestimento, especialmente a resistência à corrosão. De fato, testes com a condição ótima para evitar defeitos resultou em alto nível de diluição, não sendo adequado para a soldagem de revestimentos. Novos testes estão sendo realizados para reduzir a tendência à formação de defeitos nas condições em que se obtiveram baixos níveis de diluição.

\section{CONCLUSÕES}

Com base nos resultados apresentados no presente trabalho sobre a formação de defeitos na soldagem de revestimentos resistentes à corrosão com ligas à base de níquel depositadas pelo processo TIG com alimentação de arame frio, foi possível concluir que:

- O tecimento em espiral, embora contribua significativamente para a redução da diluição, causa uma forte instabilidade ao processo, resultando na maioria dos casos em defeitos superficiais ou defeitos entre passes.

- A condição ótima para evitar a formação de defeitos entre passes identificada pelo método Taguchi foi constituída pelas seguintes combinação de fatores de controle 2-2-2-3-3, ou seja: TE-I; $\mathrm{E}_{\text {média; }}$ Liga Hastelloy C276; Gás de proteção Ar+He; Tecimento Duplo-8.

- A condição ótima para a soldagem sem defeitos resulta em alto nível de diluição não sendo indicada para a soldagem de revestimentos resistentes à corrosão.

\section{AGRADECIMENTOS}

Os autores deste trabalho agradecem ao Laboratório de Engenharia de Soldagem - ENGESOLDA (UFC) por toda infraestrutura para realização deste trabalho. Agradece também a PETROBRAS por sua colaboração para o desenvolvimento deste trabalho. Agradece ainda a CAPES pela concessão da bolsa e aos órgãos de fomento: FINEP e CNPq pelo suporte financeiro.

\section{REFERÊNCIAS}

[1] PEREPEZKO, J.H. The hotter the engine, the better. Science. v. 326, p. 1068-1069, nov. 2009.

[2] LU, K. The future of metals. Science, v.328, pp. 319-320, 2010.

[3] SILVA CC, AFONSO CRM, RAMIREZ AJ, MOTTA MF, MIRANDA HC, FARIAS JP. Aspectos metalúrgicos de revestimentos dissimilares com a superliga à base de níquel Inconel 625. Soldagem \& Inspeção 2012;17:251 $\square 63$.

[4] HODGE, F.G. The History of Solid-Solution-Strengthened Ni Alloys for Aqueous Corrosion Service. JOM Journal of the Minerals, Metals and Materials Society. v. 58, n. 9, p. 28-31, sep. 2006. 
[5] SILVA, C.C., MIRANDA, E.C., MIRANDA, H.C., MOTTA, M.F., FARIAS, J.P. Dilution Control of Weld Overlay Superalloys Using Taguchi Method. In: 31st International Conference on Ocean, Offshore and Artic Engineering - OMAE2012, 2012, Rio de Janeiro. Proceedings of 31st International Conference on Ocean, Offshore and Artic Engineering - OMAE2012. New York: American Society of Mechanical Engineers ASME, 2012. [6] PALANI, P.K., MURUGAN, N., KARTHIKEYAN, B., 2006, "Process parameter selection for optimizing weld bead geometry in stainless steel cladding using Taguchi's approach", Materials Science and Technology. v. 22, n. 10, pp. 1193-1200. [7] SIVA, K., MURUGAN, N., LOGESH, R., 2008, "Optimization of weld bead geometry in plasma transferred arc hardfaced austenitic stainless steel plates using genetic algorithm". International Journal of Advances in Manufacturing Technology, v. XX, n. XX, pp. XX-xx.

[8] TU, S.-Y., JEAN, M.-D., WANG, J.-T., WU., C.-S., 2006, "A robust design in hardfacing using a plasma transfer arc", International Journal of Advances in Manufacturing Technology, v. 27, p. $889-896$.

[9] PALANI, P. K., MURUGAN, N., 2007, "Optimization of weld bead geometry for stainless steel claddings deposited by FCAW", Journal of Materials Processing Technology, v. 190, p. 291-299.

[10] LV, S.X., XU, Z.W., WANG, H.T., YANG, S.Q., 2008, "Investigation on TIG cladding of copper alloy on steel plate", Science and Technology of Welding and Joining, v. 13, n. 1, pp. 10-16.

[11] BENYOUNIS, K.Y., OLABI, A.G., 2008, "Optimization of different welding process using statistical and numerical approaches - A reference guide", Advances in Engineering Software, v. 39, pp. 483-496.

[12] GHANI, J.A., CHOUDHURY, I.A., HASSAN, H.H., 2004, "Application of Taguchi method in the optimization of end milling parameters", Journal of Materials Processing Technology, v. 145, p. 84-92.

[13] JUANG, S.C., TARNG, Y.S., 2002, "Process parameters selection for optimizing the weld pool geometry in the tungsten inert gas welding of stainless steel". Journal of Materials Processing Technology, v. 122, p. 33-37.

[14] SILVA, C.C., MIRANDA, E.C., MIRANDA, H.C., MOTTA, M.F., FARIAS, J.P., 2010, "Um estudo sobre a contribuição da corrente e da velocidade de soldagem sobre a diluição e as características geométricas de cordões de solda depositados pelo processo TIG com alimentação de arame frio", Soldagem \& Inspeção [Submetido para publicação].

[15] MIRANDA, E.C., SILVA, C.C., MIRANDA, H.C., MOTTA, M.F., FARIAS, J.P., 2010, "Influência do tecimento no nível de diluição e na geometria do cordão de solda utilizando o processo de soldagem TIG com alimentação automática de arame frio". Anais do XXXVI Congresso Brasileiro de Soldagem, Recife, Brasil.

[16] BANOVIC S.W., DUPONT J.N., MARDER A.R., 2001, "Dilution Control in Gas-Tungsten-Arc Welds Involving Superaustenitic Stainless Steels and Nickel-Based Alloys", Metallurgical and Materials Transactions B, v. 32B, n. 12, pp. 1171-1176.
[17] SILVA, C.C., MIRANDA, E.C., MOTTA, M.F., MIRANDA, H.C., FARIAS, J.P., 2009, "Influence of arc length on dilution and weld bead geometry of Ni-based alloy using GTAW process with cold wire feed". Proceedings of 20th International Congress of Mechanical Engineering, Gramado, Brasil.

[18] MIRANDA, E.C., SILVA, C.C., MIRANDA, H.C., MOTTA, M.F., FARIAS, J.P., 2009, “Ajuste dos parâmetros de alimentação de arame na soldagem com processo TIG para aplicações de revestimento", Anais do XXXV Congresso Brasileiro de Soldagem, Piracicaba, Brasil.

[19] PHADKE, M.S. 1989, "Quality Engineering Using Robust Design". New York, USA, Prentice Hall.

[20] MENZEL, M., 2003, "The influence of individual components of an industrial gas mixture on the welding process and the properties of welded joints". Welding International. v. 17, n. 4, pp. 262-264.

[21] LINDSTRÖM, J., RUNNERSTAM, O. 2000, “Adding NO to the Argon or Argon/Helium mixture does the trick". Svetaren, n. 2, pp. 14-16. 